Ann. Biol. anim. Bioch. Biophys., 1979, 19 (3 B), 709-714.

\title{
Mise en évidence d'une perméabilité séro-muqueuse au saccharose du jéjunum de rat
}

par Hoang Huan PHAN, Nin KHIEU

Laboratoire de Physiologie Métabolique ef Nufrition, Université de Paris VI, 9, quai Saint-Bernard, 75230 Paris Cedex 05.

Summary. Evidence for rat jejunum seromucosal permeability (SMP) to sucrose.

Intestinal SMP to sucrose has been studied by an in vivo technique using a weakly decreased gradient of sucrose concentration between the serosal and mucosal faces of a temporary Thiry-Vella loop. This gradient was carried out by introducing a sucrose load at the penis dorsal vein and by perfusing the intestinal loop with bidistilled water. Sugars present in exit perfusates proved intestinal SMP to sucrose. This SMP showed a marked decrease after the first hour of perfusion. In order to determine whether SMP was caused by the effects associated with a higher blood sucrose level or a smaller extra-cellular distribution of the sugar, intestinal resection and partial hepatectomy were performed before the experiment. No significant change in SMP level was noted. When similar experiments were attempted with glucose or fructose, it was found that SMP to these sugars presented the same characteristic change, i.e. weakly restricted permeability after the first hour of intestinal perfusion. Renal pedicles were ligated at both sides to maintain a high blood sugar concentration after sucrose intravenous injection. A sucrose load was then injected immediately or $1 \mathrm{hr}$ later concomitantly with perfusion. The results showed that the drop in SMP to sucrose after the first hour of intestinal perfusion was not a direct effect of blood sugar depletion. This suggested in situ, outwardly-directed permeability regulation at the mucosal site of the intestine.

L'hydrolyse supraluminale des disaccharides ingérés en hexoses directement absorbables est un processus normal de la digestion intestinale. Cependant, une petite quantité de ces disaccharides peut, dans certaines conditions, échapper à l'action des hydrolases, traverser la paroi intestinale par un processus de diffusion passive, puis passer dans l'urine sous leur forme originelle (Menzies, 1974 ; Larker et Menzies, 1977). II n'existe pratiquement pas d'expérience « in vivo » mettant directement en évidence leur absorption à un niveau connu de l'intestin. La recherche de ces dissacharides dans le sang mésentérique est en effet très délicate tant par leur faible quantité que par leur disparition très rapide sous l'action de la clearance rénale.

Par contre, l'éłude de la perméabilité séro-muqueuse de l'intestin aux disaccharides, processus inverse et corollaire de leur absorption, est plus facile à réaliser. On peut parfaitement délimiter un segment intestinal et récolter par perfusion les sucres ayant passé dans le milieu intraluminal sous l'effet d'un gradient de concentration. Ces 
conditions expérimentales peuvent être réalisées chez l'animal vivant par une surcharge intraveineuse en saccharose qui sera effectuée en même temps qu'une perfusion intestinale avec une solution exempte de sucre. Pour cette étude, le saccharose présente un double avantage. II n'est pas métabolisé à l'intérieur de l'organisme alors que dans la lumière intestinale, il est susceptible d'être hydrolysé par la saccharase en glucose et en fructose. Les possibilités de détecter le passage du saccharose à travers la paroi de l'intestin sont ainsi augmentées par la présence de trois sucres dans le perfusat.

Nous allons dans un premier temps mettre en évidence le phénomène de perméabilité séro-muqueuse de l'intestin, puis en étudier quelques caractéristiques mécanistiques, et enfin essayer de donner une signification fonctionnelle à ce phénomène.

\section{Matériel et méthodes.}

Après un jeûne de $16 \mathrm{~h}$, des rats Wistar mâles pesant $250 \mathrm{~g}$, porteurs de l'anse de Thiry-Vella «temporaire » (jéjunum proximal $=20 \mathrm{~cm}$ ) reçoivent une perfusion intestinale en circuit ouvert avec de l'eau bidistillée selon la technique habituelle (sous anesthésie au Nembutal, $4,5 \mathrm{ml} / \mathrm{h}$ pendant $4 \mathrm{~h}$ : débit permettant une récolte de liquide suffisante sans distension intestinale, Phan, 1971). Par ailleurs, une injection de $1,5 \mathrm{ml}$ d'une solution de saccharose à $200 \mathrm{mg} / \mathrm{ml}$ est pratiquée au niveau de la veine dorsale de la verge. Elle marque le débuł de la perfusion. Le perfusat sorti est collecté dans un tube à hémolyse gradué maintenu à $4^{\circ} \mathrm{C}$ et changé toutes les heures. L'eau bidistillée utilisée pour la perfusion présente l'avantage de faire intervenir dans de faibles limites le pouvoir tampon du jéjunum pour l'amener au $\mathrm{pH} 6,2$ constant à la sortie de l'anse intestinale (Phan, 1971). Les sucres contenus dans le perfusat sont mesurés à l'aide des techniques enzymatiques appropriées : saccharose (Dahlquist et Borgström, 1965), glucose (Huggett et Nixon, 1957) et fructose (Klotzch et Bergmeyer, 1965). Ces glucides sont d'autre part identifiés par chromatographie en couche mince (Cotte, Mathieu ef Collombel, 1964).

\section{Résultats et discussion.}

- Existence de la perméabilité séro-muqueuse du jéjunum.

Les résultats consignés dans le tableau 1 montre que l'eau de perfusion à la sortie de l'anse de Thiry-Vella contient différentes quantités de saccharose, de glucose et de fructose. La nature de ces glucides est également confirmée par chromatographie en couche mince. Il existe ainsi une perméabilité séro-muqueuse de l'intestin au saccharose. On constate qu'elle diminue en fonction du temps. C'est au cours de la première heure de perfusion que le saccharose se retrouve en plus grande quantité dans le perfusat. Ensuite, il diminue de plus de 5 fois à la deuxième heure, puis varie très peu aux heures suivantes. Le glucose et le fructose qui sont les produits de l'hydrolyse du saccharose intraluminal, accusent une baisse seulement de 2 fois entre les deux premières heures (tabl. 1 : A). Ainsi les quantités de saccharose, de glucose el de fructose présentes dans le perfusat de première heure atteignent respectivement 78, 50 et 62 p. 100 de leur quantité totale récoltée au cours de $4 \mathrm{~h}$ de perfusion. Par contre, les quantités 
de sucres existant dans le perfusat à la fin de l'expérience sont très faibles et coïncident avec une concentration sanguine insignifiante de saccharose $(0,013 \mathrm{~g} / \mathrm{l})$.

\section{TABLEAU 1}

Quantités de sucres récoltées dans les différents perfusats

\begin{tabular}{|c|c|c|c|c|c|}
\hline \multicolumn{2}{|c|}{ Heure de perfusion } & \multirow{2}{*}{ 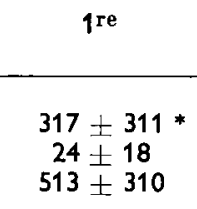 } & \multirow{2}{*}{$\begin{array}{c}2^{\mathbf{e}} \\
58 \pm 72 \\
12 \pm 9 \\
254 \pm 214\end{array}$} & \multirow{2}{*}{$\begin{array}{c}3 e \\
18 \pm 22 \\
11 \pm 10 \\
44 \pm 38\end{array}$} & \multirow{2}{*}{$\begin{array}{c}4 \mathrm{e} \\
\\
\\
12 \pm 13 \\
9 \pm 6 \\
23 \pm 27\end{array}$} \\
\hline$(n=10)$ & $\begin{array}{l}\text { Saccharose } \\
\text { Glucose } \\
\text { Fructose }\end{array}$ & & & & \\
\hline$(n=6)$ & $\begin{array}{l}\text { Saccharose } \\
\text { Glucose } \\
\text { Fructose }\end{array}$ & $\begin{array}{c}399 \pm 237 * \\
57 \pm 51 \\
672 \pm 267\end{array}$ & $\begin{array}{c}49 \pm 18 \\
15 \pm 14 \\
286 \pm 190\end{array}$ & $\begin{array}{l}21 \pm 4 \\
18 \pm 15 \\
47 \pm 31\end{array}$ & $\begin{array}{l}27 \pm 18 \\
20 \pm 14 \\
42 \pm 20\end{array}$ \\
\hline $\begin{array}{c}C \\
(n=6)\end{array}$ & Glucose & $355 \pm 323 *$ & $31 \pm 28$ & $20 \pm 17$ & $11 \pm 14$ \\
\hline$(n=6)$ & $\begin{array}{l}\text { Glucose } \\
\text { Fructose }\end{array}$ & $\begin{array}{c}140 \pm 104 \\
1717 \pm 370 *\end{array}$ & $\begin{array}{c}55 \pm 43 \\
214 \pm 149\end{array}$ & $\begin{array}{l}26 \pm 15 \\
45 \pm 19\end{array}$ & $\begin{array}{c}20 \pm 11 \\
0\end{array}$ \\
\hline$(n=10)$ & $\begin{array}{l}\text { Saccharose } \\
\text { Glucose } \\
\text { Fructose }\end{array}$ & $\begin{array}{l}707 \pm 605^{*} \\
115 \pm 116 \\
771 \pm 228\end{array}$ & $\begin{array}{c}81 \pm 42 \\
23 \pm 15 \\
361 \pm 135 \\
\end{array}$ & $\begin{array}{r}34 \pm 16 \\
16 \pm 13 \\
196 \pm 93 \\
\end{array}$ & $\begin{array}{r}35 \pm 19 \\
15 \pm 10 \\
163 \pm 69 \\
\end{array}$ \\
\hline$(n=22)$ & $\begin{array}{l}\text { Saccharose } \\
\text { Glucose } \\
\text { Fructose }\end{array}$ & $\bar{z}$ & $\begin{array}{c}58 \pm 35 \\
16 \pm 11 \\
212 \pm 117\end{array}$ & $\begin{array}{c}29 \pm 12 \\
10 \pm 6 \\
136 \pm 78\end{array}$ & $\begin{array}{r}30 \pm 24 \\
12 \pm 10 \\
123 \pm 75\end{array}$ \\
\hline
\end{tabular}

Les résultats sont des moyennes $(\mu \mathrm{g} / \mathrm{h} / 20 \mathrm{~cm}$ d'intestin) suivies de leur écart-lype. Le nombre d'animaux $n$ est indiqué entre parenthèses. Le test t est pratiqué pour les sucres utilisés en surcharge : * pour $p<0,05$ entre la quantité de sucre récoltée au cours d'une heure par rapport à celle obtenue à l'heure suivante. $A=$ surcharge en saccharose chez les rats témoins. $B, C$ ou $D=$ surcharge en saccharose, en glucose ou en fructose chez les rats ayant subi une résection intestinale et une hépatectomie partielle. $E$ ou $F=$ surcharge en saccharose immédiate ou différée de une heure par rapport au début de la perfusion intestinale chez les rats ayant les reins ligaturés. (Les grandes valeurs des écarts-types résulteraient d'une réduction plus ou moins rapide et plus ou moins prononcée de la perméabilité chez différents animaux.)

Le mécanisme impliqué dans cette perméabilité serait un processus de diffusion passive comme il a été constaté pour l'absorption intestinale des disacharides intacts (Larker et Menzies, 1977). A priori, le passage du saccharose à travers la paroi capillaire s'effectuerait librement puisque la perméabilité capillaire existe pour des substances dont le rayon moléculaire peut afteindre 12 à $24 \AA$ (Yablonski et Lifson, 1976), c'est-à-dire bien supérieur à celui du saccharose. Le passage séro-muqueux du saccharose à travers la paroi intestinale se ferait ensuite par la voie paracellulaire sans système transporteur spécifique. Cette voie représente selon Kingham, Whorwell et Loehry (1976) un complexe ae jonctions intercellulaires qui établit la communication entre la face muqueuse et la face séreuse de l'intestin par des pores situés au niveau des villosités intestinales et à la surface de la membrane latérale de l'entérocyte. Elle se prête à un fluide bidirectionnel qui existe bien au niveau du jéjunum de rat (Munck 
et Rasmussen, 1977). La voie paracellulaire semble donc répondre à la perméabilité séro-muqueuse de l'intestin au saccharose.

\section{- Propriétés de la perméabilité séro-muqueuse du jéjunum.}

a) Limite de perméabilité.

Pour étudier la variation de la perméabilité séro-muqueuse du jéjunum au saccharose, nous avons recours à deux procédés qui permettent d'augmenter la concentration du saccharose circulant sans modifier la quantité de saccharose administrée $(300 \mathrm{mg})$. Le premier procédé est une résection intestinale massive ne laissant subsister que l'anse de Thiry-Vella et $3 \mathrm{~cm}$ de duodénum proximal nécessaire à l'écoulement de la bile et des sécrétions pancréatiques. Le passage séro-muqueux du saccharose à travers la paroi intestinale se fait alors principalement au niveau de l'anse perfusée. Le deuxième procédé est une hépatectomie partielle enlevant les $2 / 3 \mathrm{du}$ foie et réduisant dans les mêmes proportions les espaces extracellulaires et le volume sanguin enfermés dans les lobes excisés.

Contrairement à toute attente, la surcharge intraveineuse en saccharose chez les rats ayant subi cette double opération montre que les quantités de sucres récoltées dans les perfusats ont des valeurs voisines de celles observées précédemment (tabl. $1:$ B). En particulier, la perméabilité séro-muqueuse au saccharose subit également une forte restriction à la fin de la première heure de perfusion. Or, les ablations d'organes ont bien provoqué une augmentation de saccharose circulant, augmentation qui s'est traduite par un accroissement de 29 p. 100 de l'excrétion urinaire de ce disaccharide. Ainsi la perméabilité séro-muqueuse du jéjunum n'est pas modifiée lorsque l'afflux du saccharose devient momentanément plus important. A une surcharge donnée, elle semble avoir une limite maximale qui ne varie pas de façon continue comme une simple diffusion passive.

\section{b) Spécificité de la perméabilité séro-muqueuse.}

Dans les expériences précédentes nous avons pu remarquer que les quantités de glucose et de fructose suivent étroitement celle du saccharose. II serait intéressant d'examiner si la présence de ces hexoses est due uniquement à l'hydrolyse du saccharose, et de leur absorption intestinale incomplète. Ainsi le glucose et le fructose sont utilisés en quantité équimoléculaire à la place du saccharose.

Les résultats obtenus montrent que sous l'effet d'un gradient de concentration, le glucose et le fructose traversent la paroi intestinale dans le sens séro-muqueux de la même manière que le saccharose. Après la première heure de perfusion, leur passage dans la lumière intestinale se réduit fortement : de 11 fois pour le glucose et de 8 fois pour le fructose (tabl. $1: C$ et D), diminution que l'on pourrait attribuer à une baisse rapide de leur concentration sanguine. Certes, le glucose et le fructose introduits par voie intraveineuse, sont rapidement métabolisés (Heuckenkamp et Zollner, 1972). Toutefois, l'hépatectomie enlevant les $2 / 3$ du foie, la conversion métabolique du fructose en glucose (MacDonald et Turner, 1968) et la résorption rénale active du glucose font que la concentration sanguine du glucose reste assez élevée à la fin de l'expérience (par exemple, fructosémie nulle et hyperglycémie de $1,05 \mathrm{~g} / \mathrm{l}$ dans le cas de surcharge en fructose). 
Quoi qu'il en soit, la perméabilité séro-muqueuse de l'intestin est un processus qui concerne également les nutriments glucose ef fructose à l'encontre de leur rapide absorption intestinale.

c) Régulation de la perméabilité séro-muqueuse de l'intestin.

On peut se demander si la diminution de la concentration sanguine des glucides sous l'action de la clearance rénale suffit à expliquer la forte restriction de la perméabilité séro-muqueuse intestinale constatée après la première heure de perfusion. Quelle sera l'évolution de cette perméabilité si l'on supprime l'émonctoire rénal immédiatement après la surcharge en saccharose?

Les résultats consignés dans le tableau (1: E) montrenł que les quantités de sucres récoltées dans les perfusats obtenus chez les rats ayant subi une surcharge en saccharose puis les ligatures rénales, présentent aussi une forte diminution après $1 \mathrm{~h}$ de perfusion malgré qu'il existe une saccharémie élevée à la fin de l'expérience $(1,143 \mathrm{~g} / \mathrm{l})$.

Dans ce cas, la quantité de saccharose récoltée dans le perfusat de première heure représenterait, par rapport à celles faibles et peu variées notées aux 3 dernières heures de perfusion, une augmentation de perméabilité qui serait favorisée par une vacuolisation réversible de l'épithélium intestinal induite par l'afflux de saccharose, comme l'ont observé Monserrat et Chandler (1975) au niveau de l'épithélium du tube contourné rénal.

Pour vérifier cette éventualité, nous avons différé de 1 h la surcharge en saccharose après avoir établi la perfusion intestinale et les ligatures rénales. Le sang est alors surchargé en saccharose à partir de la $2^{e}$ heure de perfusion. Les quantités de sucres récoltées dans les perfusats sont faibles pour les 3 dernières heures de perfusion (tabl. $1:$ F) et restent comparables à celles observées après la 1 re heure de perfusion dans les autres types d'expérience. Ainsi, indépendamment de tout effet exercé par l'afflux d'une quantité élevée de saccharose, la perméabilité séro-muqueuse de l'intestin diminue fortement après une heure de perfusion intestinale.

En résumé, il existe une perméabilité séro-muqueuse du jéjunum au saccharose, au glucose et au fructose. Elle présente une limite maximale sous l'effet d'une surcharge intraveineuse en glucide, se réduit, indépendamment de la concentration sanguine, de 5 à 11 fois après qu'une absorption intestinale d'eau s'est établie. La fuite des nutriments glucidiques vers la lumière intestinale serait donc très réduite mais suffisante au niveau du glycoalyx pour assurer une activité basale des osidases en dehors des périodes de digestion.

Commission CNERNA Digestion-Absorption/Association des Physiologistes, Paris 5-6 octobre 1978.

Remerciements. - Ce travail a bénéficié de l'aide financière du contrat de recherche libre de I'INSERM no 77.1.188.7.

\section{Références}

COTTE J., MATHIEU M., COLLOMBEL C., 1964. Méthodes d'identification des sucres sanguins ef urinaires par chromatographie en couche mince. Path. Biol., 12, 747-749.

DAHLQVIST A., BORGSTRÖM B., 1964. Digestion and absorption of disaccharides in man. Bioch. J., $7,18-25$. 
HEUCKENKAMP P. U., ZOLLNER N., 1972. The comparative metabolism of carbohydrates administered intravenously. Nutr. Metab. (Suppl.), 14, 58-73.

HUGGETT A. St G., NIXON D. A., 1957. Use of glucose oxidase, peroxidase and o-dianisine in determination of blood and unary glucose. Lancet, 2, 236.

KLOTZCH N., BERGMEYER H. U., 1965. D-Fructose, 156-159. In BERGMEYER H. U., Methods of enzymatic analysis. Acad. Press N. Y.

KINGHAM J. G. C., WHORWELL P. J., LOEHRY C. A., 1976. Small intestinal permeability. Gut, 17, 354-361.

LARKER M. F., MENZIES I. S., 1977. Increase in human intestinal permeability following ingestion of hypertonic solutions. J. Physiol., 265, 881-894.

MACDONALD I., TURNER L. J., 1968. Serum fructose levels after sucrose or its constituent monosaccharides. Lancet, 1, 841-843.

MENZIES I. S., 1974. Absorption of intact oligosaccharides in health and disease. Bioch. Soc. Trans., 2, 1042-1047.

MONSERRAT A. J., CHANDLER A. E., 1975. Effects of repeated injections of sucrose on the kidney. Virchows Arch. B Cell Path., 19, 77-91.

MUNCK B. G., RASMUSSEN S. N., 1977. Paracellular permeability of extracellular space markers across rat jejunum in vitro. Indication of a transepithelial fluid circuit. J. Physiol., 271, 473-488.

PHAN H. H. 1971. Influence de l'acide pantothénique sur l'absorption intestinale du glucose et son devenir chez le rat. Thèse Doct. ès Sc. (Paris), $160 \mathrm{pp}$.

YABLONSKI M. E., LIFSON N., 1976. Mechanism of production of intestinal secretion by elevated venous pressure. J. clin. Invesi., 57, 904-915. 